\title{
Research on Multidisciplinary Design Modeling of LED Lighting
}

\section{Products}

\author{
Feng Xiong ${ }^{1, a}$, Renfei Ma ${ }^{1, a^{*}}$, Jianjian Mei ${ }^{1, a}$, Lilan Liü ${ }^{1, a}$, Tao $\mathrm{Yu}^{1, \mathrm{a}}$ \\ ${ }^{1}$ Cims \& Robot Center, Shanghai University, Shanghai, China \\ a2482428602@qq.com
}

Keywords: Multidisciplinary modeling; a process; design object tree; the performance function tree

\begin{abstract}
LED lighting products play a very important role in energy conservation and environmental protection. For the multidisciplinary design problem of LED lighting products, we establish the information model of LED lighting products based on the object tree, that is, a process and two trees. First we build a multidisciplinary design process of LED lighting products, clearing tasks and indicators of product design. Then we discuss the LED product modeling which is based on the design object tree and construct two trees of LED products, providing a necessary theoretical support for the design of LED lighting products. Finally, through the example of application, verify the feasibility of multidisciplinary design modeling.
\end{abstract}

\section{Introduction}

LED is a new solid cold light source. It has features of high energy efficiency, long life, low voltage, simple structure, small size, light weight, fast response, good seismic performance, and full color spectrum $^{[1]}$. Especially in terms of energy saving and environmental protection, LED lighting products have a distinct advantage compared with ordinary incandescent and fluorescent lamps. It makes them widely applied on various occasions. LED lighting is the current emerging industry which is supported by our country. To promote energy conservation and achieve sustainable development, our country is phasing out incandescent lamps. It is the market demand for LED lighting products that promote the vigorous development of LED lighting products.

On the other hand, while our country has many LED lighting enterprises, most of them are lack of core technology. They are mainly in the LED industry chain downstream. They are engaged in the processing and assembly of LED products. The quality of LED products is often uneven. This is because LED lighting products are involved in many disciplines. Through the study of LED products, it can be subdivided into the optical, electrical, thermal and multidisciplinary and many other disciplines. Various disciplines are involve in the material and device development, optical design, structural design, packaging materials, circuit design, lighting development, lighting effects , visual matching and so on. It belongs to the high-tech field of multidisciplinary and is a typical multidisciplinary design problem ${ }^{[2]}$. To solve this problem, we need to work on the research of multidisciplinary design of LED lighting products modeling to help designers to express the exact ideas of design and improve the convenience of modeling. Thereby improve LED lighting products performance and reliability. 


\section{The basis of multidisciplinary design modeling of LED lighting products}

The abstraction of LED lighting products at different levels ( such as a process, a structure or a certain type of performance of the design) is called design object. And the behavior and method of describing design objects is called design method. Product modeling is to describe the relationship between design objects ${ }^{[3]}$.

A complete model of multidisciplinary products includes: a complete design process; each stage of the design process has its design object tree; each node in the design object tree has its corresponding performance function tree. Therefore the main content of the multidisciplinary design modeling of LED lighting products is summarized as "a process and two trees."

Next, for lighting products related to several concepts, we make an explanation:

1. A process actually refers to the product design process. Establish the mapping from the problem space (function space) to the solution space (property space ${ }^{[4]}$.Each stage of the design process has design object tree and performance function tree.

2. The design object tree refers to a tree which is formed by product systematic structure. That is the structure of the product components to achieve a certain function.

3. The performance function tree refers to a tree structure which is formed by the product performance. It is also required by the products and satisfies the relationship between the design requirements and other attributes.

4. Each node of the design object tree is a design object. The description of design objects mainly includes three basic elements: design goals, design constraints, design variables (Parameters).

For LED lighting products, its product modeling process is shown in Fig. 1.

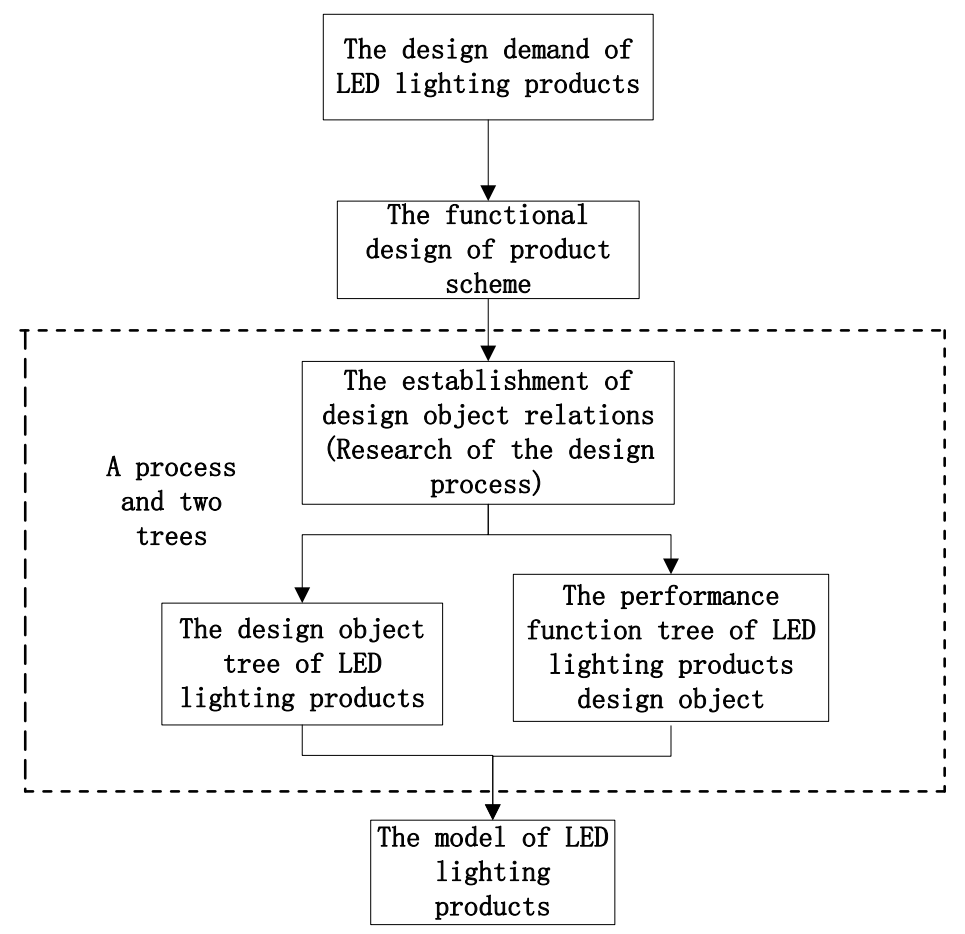

Fig.1 The process of establishing the model of LED lighting products.

Based on this process, I do the following research for "a process" and "two trees" of the LED lighting products multidisciplinary design modeling. 


\section{The multidisciplinary design modeling process of LED lighting products}

Establishment of LED lighting products multidisciplinary design process. For this study of LED lighting product modeling, it proposes "a process". According to the design characteristics of most LED lighting products, the design process is shown in Fig.2. Of course, the system can be adjusted according to the specific product.

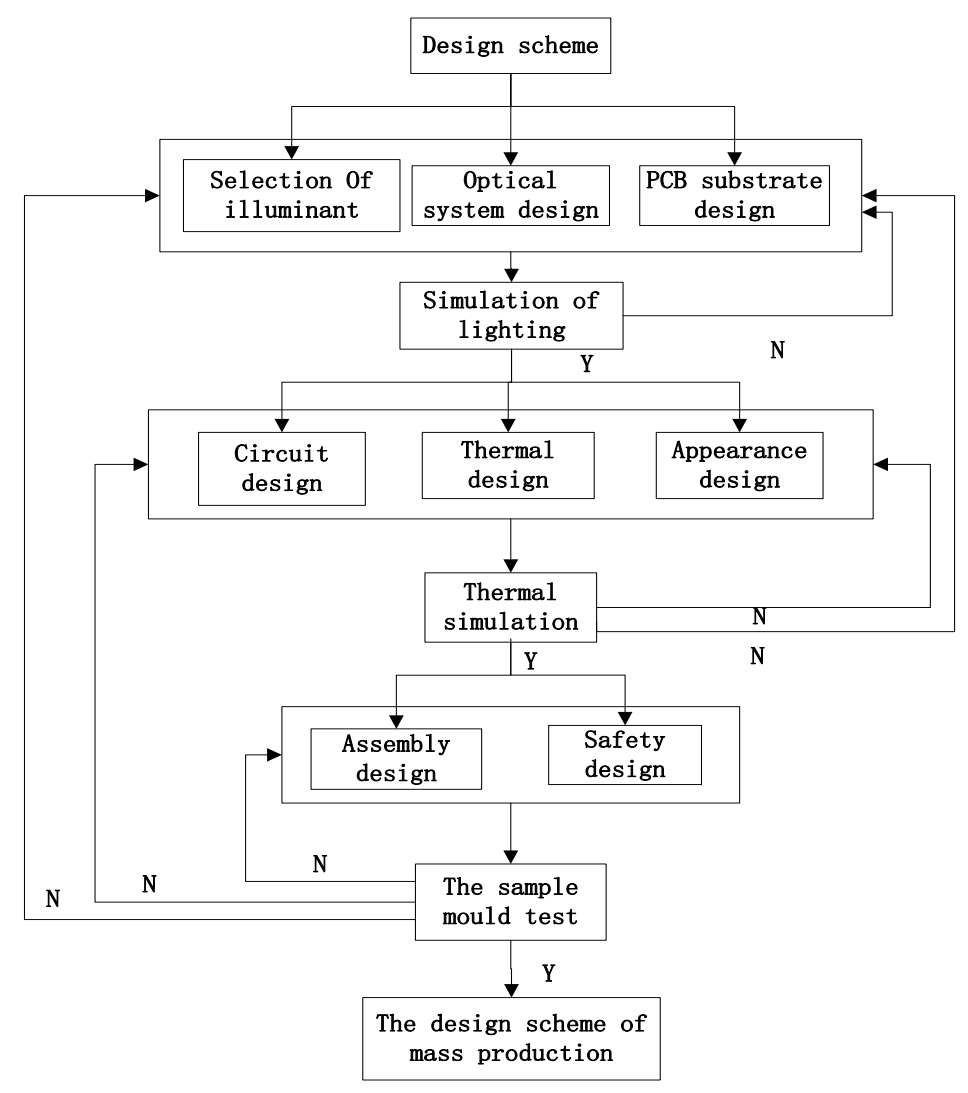

Fig. 2 The design process of LED lighting products with parameters.

The design process are described below:

1. First, according to the optical requirements of products(flux, power, light angle, etc.),select the light source. Determine the brand and the number of chips according to the power. Design the optical system according to the arrangement of the chip. With circuit design, design chip circuit on the board. And determine whether the task can be achieved by light efficiency indicators. Then determine the next design. ${ }^{[5]}$

2. After the optical design is completed, set out to circuit design, thermal design and housing design. Work on drive design or selection with circuit design. If it has control requirements, we need to work on controller design. With the distribution of the chip on the board and total power design parameters of the radiator, calculate the thermal simulation analysis. Depending on whether the temperature distribution of the product meets mission targets, we determine the next design. ${ }^{[6]}$

3. Finally, according to the assembly design of product and safety design and so on, determine the whole lamp design of LED lighting products. ${ }^{[7]}$

From chart analysis, We need to modify the parameters repeatedly in the detailed parameters design phase. At this point, we conducted a multidisciplinary design modeling which is very important. It can improve the efficiency of design.

LED product modeling based on product design object tree. For the multidisciplinary modeling of LED lighting products, because of the diversity of product usage, product type 
diversification and customization needs, it is very important to establish appropriate product design object tree to make product model flexible enough.

In the composition of the multidisciplinary model of LED products and except the presence of serial and parallel relationship between the design process, the relationship between product design objects has hierarchical tree structure relationship, no matter what is from the product system-wide structure (regardless of function or structural components to achieve function and other cell structures) to consider, or is considered from the full performance of the product contained (the performance is as a design object $)^{[8]}$. Any node in the hierarchy tree are a design object which has its own object properties. For the optimal design of existing products, it is relatively easy to generate the object tree. In establishing the object tree of the product and determining node elements, it can be done in the following three steps:

Step one: Generate the design object tree frame. According to "the whole lamp - components parts" relationship of LED products, draw a sketch of the design object tree. Depending on the difference of the LED type and design type, establish the appropriate framework for the object tree.

Step two: Determine the design object parameters. Analyze and classify all the parameters of components or parts. Extract such as geometry parameters, technical parameters, material parameters and performance parameters and so on. Put it into corresponding sub-node of design objects.

Step three: Determine the performance function. Establish performance function of each node of the product design object tree.

Through the above steps, construct two trees of LED lighting product model. It is shown in Fig. 3. 


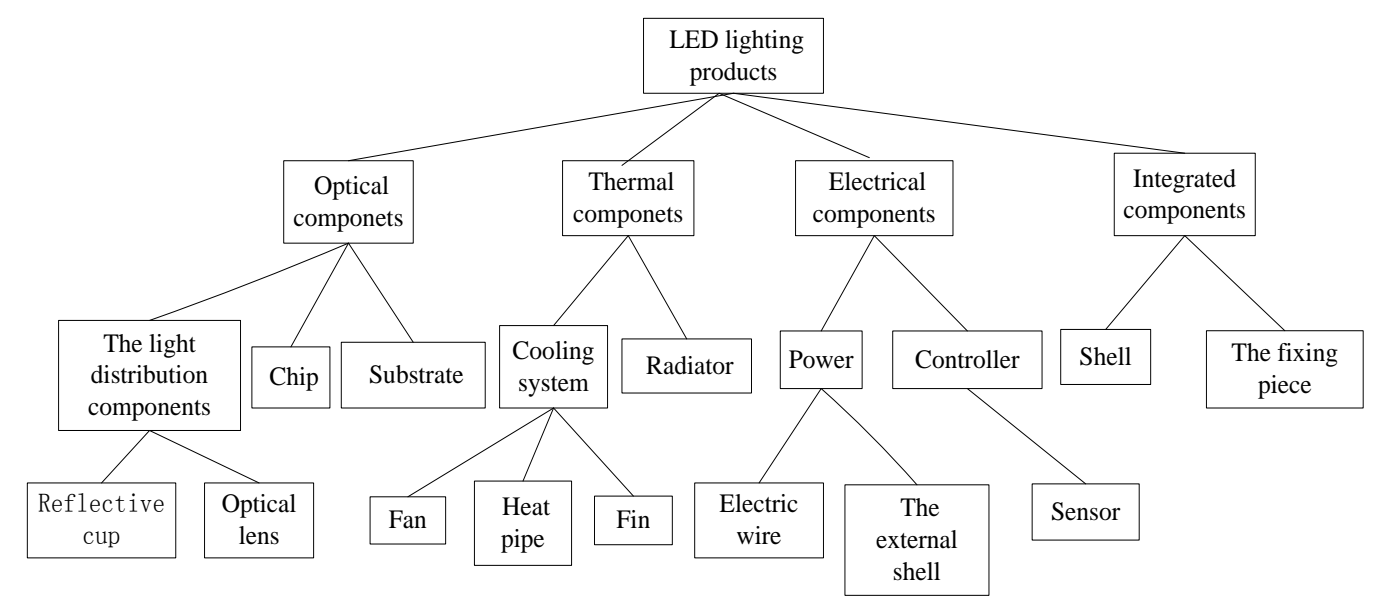

(a) Product design object tree

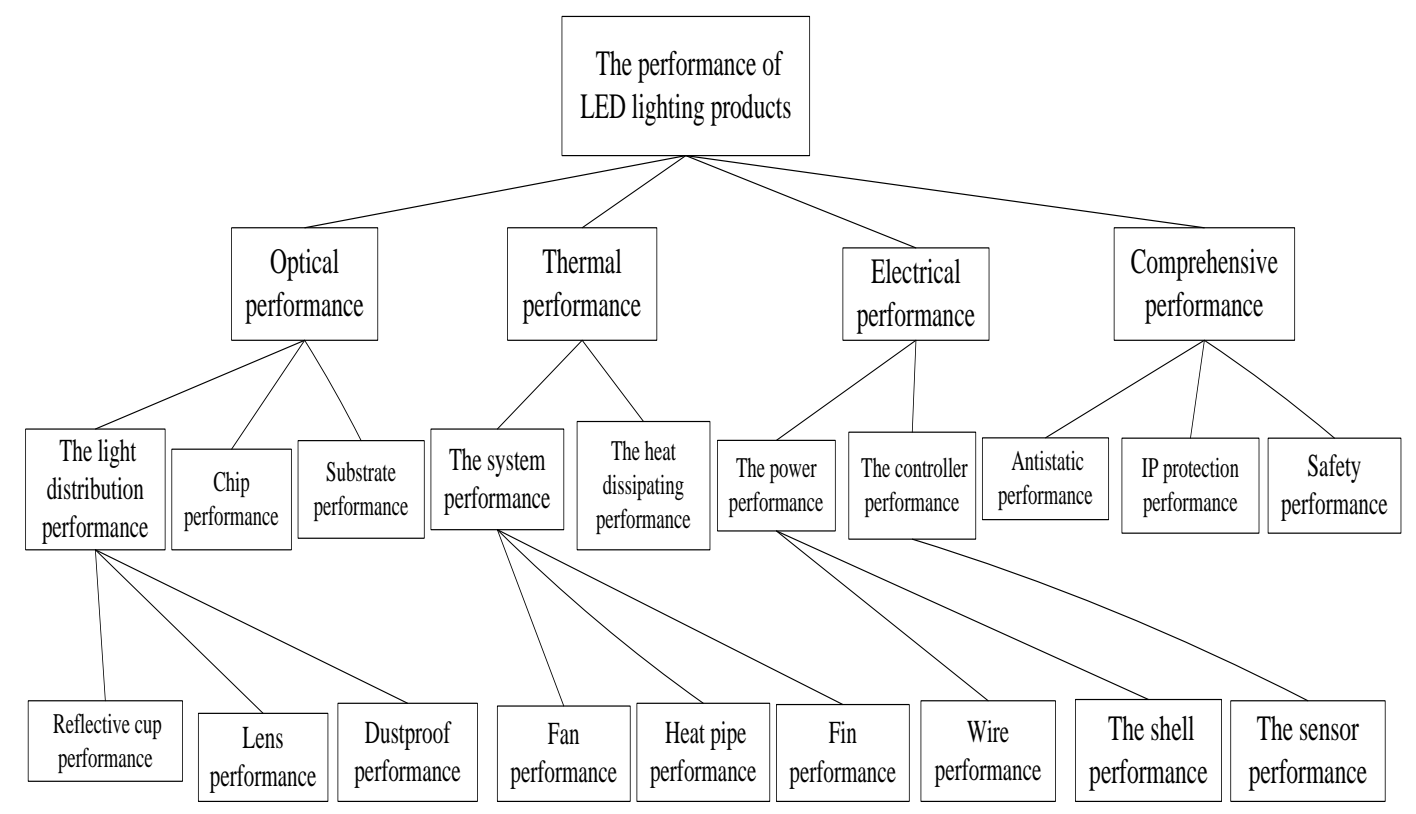

(b) The performance function tree

Fig .3 The two trees' structure of LED lighting products.

\section{Applications for examples}

Based on its own design and development of an MR16 LED spotlights, set out to make multidisciplinary product design modeling.

According to the steps illustrated in Figure 3, establish the mapping relationship between MR16 LED spotlight product design object tree and the performance function tree shown in Figure 4.As shown in Figure 4, products, components, parts-level nodes constitute a design object tree frame of LED Spotlight products. Each node is a design object. The system level is the whole lamp LED spotlight.Component level is divided into optical components, power control unit, cooling components, integrated components..Analogy other parts level node in this manner. The property level is on the far right. The property level variables, including design objects, performance function and design object model. Finally, through Figure 3, establish the whole lamp design object tree and performance function tree of LED spotlights as shown in Figure 4.From modeling results, modeling and analysis can be carried out by the above method for any type of LED lighting products. 


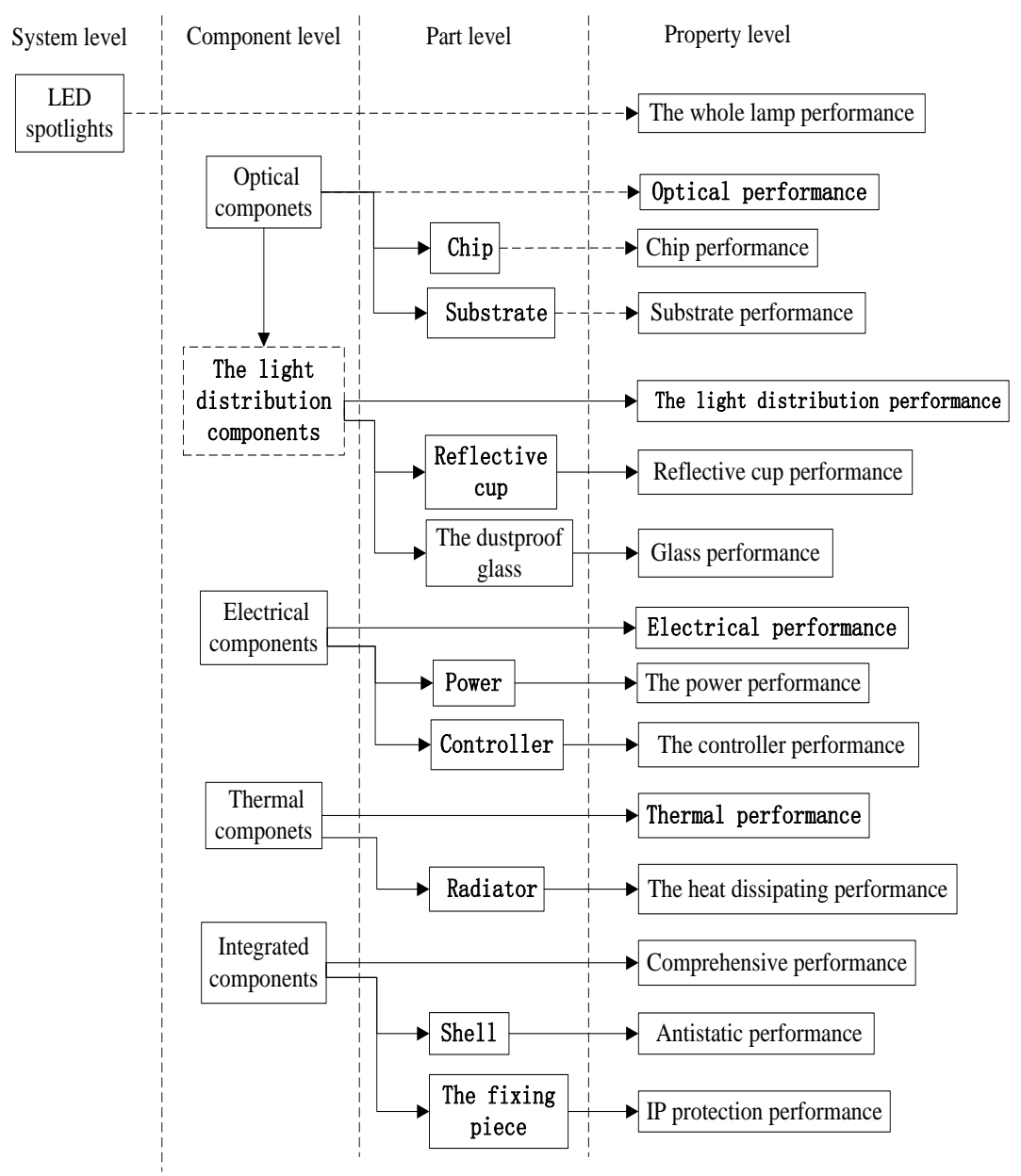

Fig. 4 The design object tree and the performance function tree of LED spotlight.

\section{Conclusion}

Through the research of theoretical modeling of LED lighting products, establish the model of the LED lighting product based on the object tree. That is "a process" and "two trees". This can help designers to articulate design ideas, greatly reducing product design blindness and repeatability which effectively improves work efficiency and quality. In a certain extent, this solves the problems of the multidisciplinary LED lighting product design and enhances product performance and the company's core competitiveness.

\section{Acknowledgement}

This work is supported by National Science and Technology Support Program (NSTSP) under Grant no. 2013AA03A112. The authors are grateful for the financial support.

\section{References}

[1] Peimin Fang, The development situation of LED lighting lamp, Electronic Products. (2007) 1-2.

[2] Ali Serpenguzel, Solid state lamp: The new paradigm in illumination, World Congress on Housing Process and Produce(2003).

[3]Yifang Zhong, Baihong Chen, Zhouhong Wang, The principle and method of the multidisciplinary integrated optimization design, Huazhong University of Science and Technology press, Wuhan,2006,pp.26-39. 
[4] Liang Chen, Guodong Jin, The Product Modeling System of Multidisciplinary Collaborative Design in Distributed Network Environment, Machine Tool \& Hydraulics. (2006) 1-2.

[5] Haiming Fang, LED lighting design and case selection, Beihang University Press, Beijing, 2012.

[6] Haiming Fang, LED lighting and engineering design examples, China Electric Power Press, Beijing, 2012.

[7] Zuming Liu, Xiaotao Li, LED lighting design and application, Publishing House Of ectronics Industry,Beijing,2011.

[8]Yifang Zhong, Baihong Chen, Zhouhong Wang, The principle and method of the multidisciplinary integrated optimization design, Huazhong University of Science and Technology press, Wuhan, 2006,pp.26-39. 